\title{
Excitonic theory of stark ladder spectra in superlattices
}

\author{
D.M. WHITTAKER
}

Department of Physics, University of Sheffield, Sheffield, S3 7RH, U.K.

\begin{abstract}
Predicting exciton behaviour in the Stark ladder regime is an interesting theoretical problem, requiring the treatment of strong coupling between a number of in-plane exciton states with different values of the electron-hole separation. In this paper a new method is described which allows an essentially exact calculation of Stark ladder spectra, with negligible broadening, over a wide range of fields. The oscillator strength at each energy is evaluated from an exciton Green's function which is constructed using numerical solutions of the set of coupled differential equations corresponding to the two dimensional in-plane motion on isolated layers. When suitably broadened, the spectra realistically reproduce those obtained experimentally. At higher resolution more details can be seen, including the Fano-resonance lineshapes of the exciton peaks caused by coupling to continuum states.
\end{abstract}

\section{INTRODUCTION}

When an axial electric field is applied to a superlattice, Wannier-Stark localisation occurs, with the minibands breaking down into localised states, separated in energy by the potential dropped across a single period. Hence, in optical spectra, a Stark ladder of transitions between electron and hole states peaked in different wells is observed. At high fields, a two dimensional exciton spectrum can be associated with each such transition. However, at lower fields, when the transitions become closer together, the coupling between the exciton states becomes important. The energies and oscillator strengths of the peaks show a very complicated field dependence due to their interactions and anti-crossings. The line-shapes also become asymmetric, and it will be shown in the present paper that this is a result of resonant interactions between the (quasi) bound exciton states and the continuum states at the same energy.

The problem of the exciton in a superlattice has been considered theoretically by a number of authors [1-8]. Previous approaches have involved the expansion of the eigenfunctions of the system in some finite basis set, either plane waves [1-2] or uncoupled two dimensional exciton states [3-4], or variational calculations of a few exciton states [5-8]. Though reasonable results are obtained for the bound states, it is impossible to calculate accurate spectra using the finite basis methods because the continuum is approximated by a set of discrete peaks which need to be broadened to get realistic results. Furthermore, to improve the resolution it is necessary to diagonalise an increasingly large basis set. In the present paper an effective exciton Hamiltonian is derived and a new method of solution which gets round these problems is described. The spectra so obtained are essentially exact, and the broadening can be made arbitrarily small at little extra computational cost. 


\section{THEORY AND CALCULATION}

The electron and hole eigenstates in an unperturbed superlattice are simply Bloch states, characterised by a miniband index and their wave-vectors along and perpendicular to the growth axis. To obtain the excitonic Stark ladder states, these eigenstates need be mixed together by the perturbation due to the applied electric field and the Coulomb interaction. The approach used in this paper is to restrict the states used in constructing the exciton to a single pair of minibands (one each for electron and hole) and diagonalise the perturbation exactly within this basis. Such an approximation is usually very good, because the miniband separations in typical superlattices tend to be large compared to the energy scale of the excitonic effects.

For the present purpose, the miniband states can best be described in a representation based on localised Wannier functions [9]. The exciton wavefunction can then be written in the form

$$
\Psi\left(z_{e}, z_{h}, r\right)=\sum_{n} \psi(n, r) f_{e}\left(z_{e}-n d\right) f_{h}\left(z_{h}\right)
$$

Here, $\psi(n, r)$ is a superlattice scale 'envelope function' depending only on the separations of the electron and hole: $n$ ( $n d$, the number of periods) along the growth axis and $r$ in the plane of the wells. $f_{e}\left(z_{e}\right)$ and $f_{h}\left(z_{h}\right)$ are the Wannier functions for the pair of minibands under consideration.

In the Wannier representation, the miniband kinetic energy takes the form of a tight-binding like hopping operator:

$$
T_{\text {hop }} \psi(n, r)=\Delta_{1}[\psi(n+1, r)+\psi(n-1, r)]+\Delta_{2}[\psi(n+2, r)+\psi(n-2, r)]+\ldots
$$

where the hopping terms $\Delta_{1}, \Delta_{2}$ etc are obtained from a Fourier expansion of the joint miniband dispersion:

$$
\varepsilon_{e}(q)+\varepsilon_{h}(q)=2 \Delta_{1} \cos q d+2 \Delta_{2} \cos 2 q d+\ldots
$$

The envelope function $\psi(n, r)$ is determined by the perturbation, $V$, acting on the superlattice. For the Stark ladder, these are the Coulomb interaction and applied field, $F$, which give

$$
V\left(z_{e}-z_{h}, r\right)=-\frac{1}{\varepsilon_{r}} \frac{1}{\sqrt{\left(z_{e}-z_{h}\right)^{2}+r^{2}}}+e F\left(z_{e}-z_{h}\right)
$$

By expressing $V$ in the Wannier representation, it can be shown that the envelope function obeys a Schrödinger equation with Hamiltonian

$$
H_{e f f}=-\frac{1}{2 m} \nabla_{r}^{2}+T_{h o p}+V_{e f f}(n, r)+e F n d
$$

with $m$ the in-plane reduced mass. The effective potential is given by

$$
V_{e f f}(n, r)=-\frac{1}{\varepsilon_{r}} \int d z_{e} \int d z_{h} \frac{\left|f_{e}\left(z_{e}\right)\right|^{2}\left|f_{h}\left(z_{h}\right)\right|^{2}}{\sqrt{\left(z_{e}-z_{h}+n d\right)^{2}+r^{2}}}
$$

$V$ also has small off-diagonal terms coupling different values of $n$. However, it is easy to show that the coupling is always negligible, being typically less than $10^{-3}$ times the magnitude of the hopping terms. With this proviso, Eqn.5 is, despite its tight binding form, an exact formulation of the superlattice exciton problem for a single pair of minibands, and is valid for minibands of any width.

Optical spectra are obtained from Equ.5 using a generalisation of the method developed by Zimmermann [10] for quantum well excitons. Instead of finding eigenstates directly, the inhomogeneous system of equations

$$
\left[H_{e f f}-E\right] G\left(n, r ; n^{\prime}, r^{\prime} ; E\right)=\delta_{n, n^{\prime}} \delta^{2}\left(r^{r}-r^{\prime}\right)
$$

is solved for the Green's function $G\left(n, r ; n^{\prime}, r^{\prime} ; E\right) . \quad G$ is related to the eigenstates $\psi_{i}(n, r)$ and eigenvalues $E_{i}$ of Eqn.5 by the fundamental identity [11]

$$
G\left(n, r ; n^{\prime}, r^{\prime} ; E\right)=\sum_{i} \frac{\psi_{i}^{*}\left(n^{\prime}, r^{\prime}\right) \psi_{i}(n, r)}{E_{i}-E} .
$$




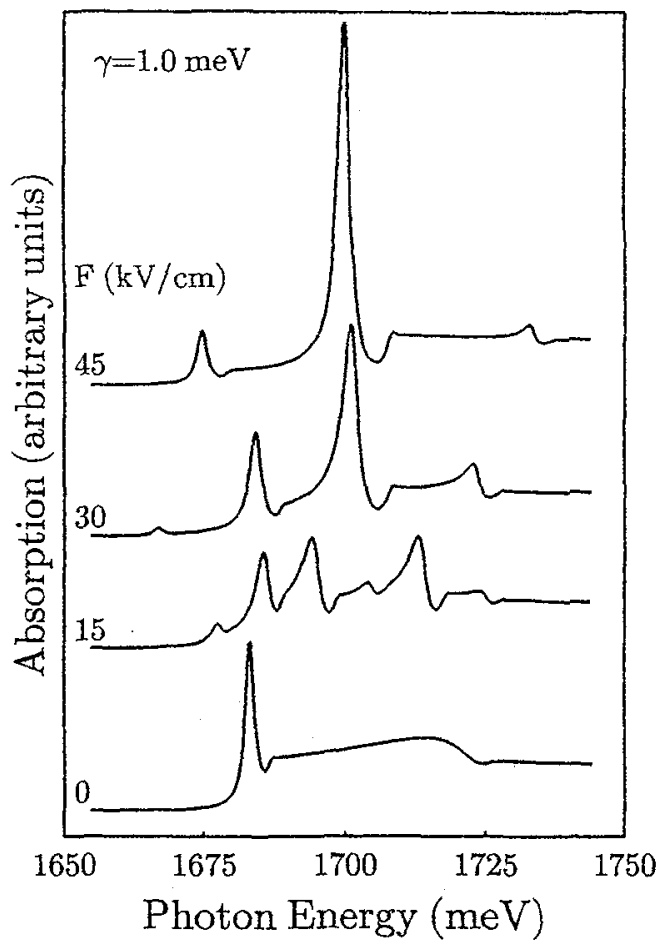

Fig.1 Stark ladder spectra in $65 \AA$ superlattice for various values of the axial field, $F$. The spectra have a Lorentzian broadening of half width $\gamma=1 \mathrm{meV}$.

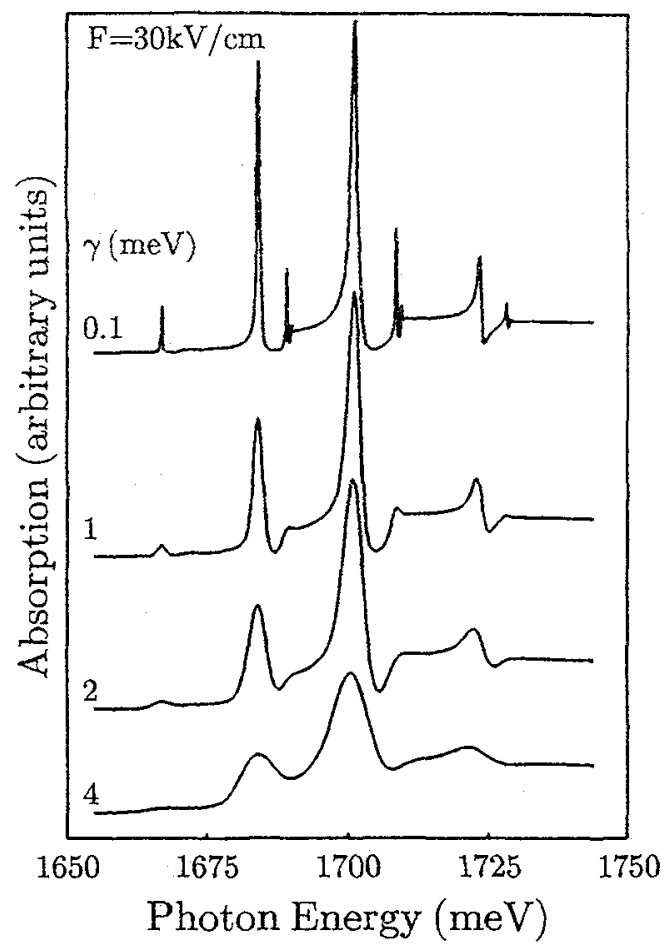

Fig.2 Stark ladder spectra in $65 \AA$ superlattice with an axial electric field of $30 \mathrm{kV} / \mathrm{cm}$ and various amounts of Gaussian broadening, half widths, $\gamma=0 \rightarrow 5 \mathrm{meV}$.

The oscillator strength at energy $E$ is thus given by the imaginary part of $\lim _{\delta \rightarrow 0} G(0,0 ; 0,0 ; E-i \delta)$.

The procedure used in the numerical solution of Eqn. 6 will only be summarised here. It is a generalisation of the standard method for solving the two point boundary value problem, in which the Green's function is constructed from solutions of the homogeneous equation, with appropriate matching at $r=r^{\prime}$ [11]. The important point is that for $r<r^{\prime}$ the solution used need only satisfy the boundary condition at $r=0$, while for $r>r^{\prime}$ only the boundary condition at $r \rightarrow \infty$ must be satisfied. As it is not necessary to satisfy both boundary conditions simultaneously, just one integration (in each direction) needs to be done.

In the present problem, the localisation of the states by the electric field means that exact results can be obtained from a finite set of, say, $N$ coupled equations, where eFNd miniband width. The $r$ range is split into two regions $\left(r^{>} \geq r^{\prime}\right)$, in each of which $G$ is expressed as a linear combination of $N$ solutions to Eqn.5 satisfying the appropriate boundary conditions. These are generated numerically by integrating the coupled system from either boundary to the matching point $r=r^{\prime}$. The coefficients in the linear combination are obtained by ensuring that the $2 N$ matching conditions imposed by the delta function at $r=r^{\prime}$ are correctly satisfied. The spectrum is calculated by following this procedure with a small values of $r^{\prime}$ and $\delta$ to obtain $\lim _{\delta \rightarrow 0} G(0,0 ; 0,0 ; E-i \delta)$ at each energy for which the oscillator strength is required.

\section{RESULTS AND DISCUSSION}

Example calculations have been carried out for a $\mathrm{GaAs} / \mathrm{Al}_{0.35} \mathrm{Ga}_{0.65} \mathrm{As}$ superlattice with $30 \AA$ wells and $35 \AA$ barriers. Such a structure has been studied experimentally by Mendez et al [12], and 
both theory and experiment give a miniband width of about $40 \mathrm{meV}$.

Fig.1 shows the calculated absorption spectra at various values of axial electric field. The results cover the range of field regimes, from high fields $(F=45 \mathrm{kV} / \mathrm{cm})$ where the localisation is essentially complete and the spectrum is dominated by transitions involving the electron and hole in the same well, down to zero field, where a single exciton peak appears below the miniband. At intermediate fields, the spectra are much more complicated due to the coupling between the many exciton peaks. Detailed theoretical discussions of this behaviour have been given by a number of authors $[2-4,7-8]$ and can be found in the references.

The spectra in Fig.1 have been broadened with a Lorentzian lineshape of half width $\gamma=1.0 \mathrm{meV}$. This is the 'natural' way to introduce broadening into the calculation, since it simply requires the addition of a finite imaginary part to the energy. In real structures the inhomogeneous broadening due to well width fluctuations is more like Gaussian in form, so to produce realistic results the spectra have to be calculated at high resolution and convoluted with a Gaussian function. Such spectra are shown in Fig.2, for a field $F=30 \mathrm{kV} / \mathrm{cm}$ and a range of values of the half width $\gamma$. For the largest broadening $(\gamma=4 \mathrm{meV})$, the spectrum is similar in form to those obtained experimentally, with just three peaks visible, corresponding to the in-plane $1 s$-like state of excitons with electronhole separations of $-1,0$ and +1 periods. As the inhomogeneous broadening is reduced, more detail becomes apparent, including the -2 state and peaks associated with excited states of the in-plane motion.

Even at the highest resolution $(\gamma=0.1 \mathrm{meV})$, the main exciton peaks still have a finite width $\sim$ $1 \mathrm{meV}$. This is actually an intrinsic feature, and arises from the mixing, by the coulomb interaction, between the bound state and continuum states at the same or nearby energies. The coupling means that there are no true bound states, only quasi-bound resonances, for which lifetimes $\sim 10^{-12} \mathrm{~s}$ can be estimated from the line width. As the field is increased, the coupling becomes weaker so the lifetime of the resonances increases.

Another consequence of this mixing is the Fano resonance line shapes apparent in the higher resolution spectra. These are characteristic of resonant interactions between bound and continuum states and consist of a peak followed by a dip in oscillator strength below the level of the surrounding continuum. Though the resolution of the available experimental spectra do not permit this sort of behaviour to be seen in detail, it explains the strongly asymmetric line shapes which are frequently observed in the intermediate field regime. Furthermore, the $\gamma=2 \mathrm{meV}$ spectrum shows that not much increase in sample quality would be required to make the Fano lineshapes readily visible.

Acknowledgment: The author wishes to thank R.Zimmermann for suggesting that the Green's function method could be applied to excitons in superlattices.

[1] Hanyou Chu and Yia-Chung Chang, Phys.Rev.B 40 (1987) 2946.

[2] S.L.Chuang, S.Schmitt-Rink, D.A.B.Miller and D.S.Chemla, Phys.Rev.B 43 (1991) 1500.

[3] D.M.Whittaker, Phys.Rev.B 41 (1990) 3238.

[4] D.M.Whittaker, Superlattices and Microstructures 7 (1990) 375.

[5] A.Chomette, B.Lambert, B.Deveaud, F.Clerot, A.Regreny and G.Bastard, Europhys.Lett. 4 (1987) 461.

[6] R.H.Yan, F.Laruelle and L.A.Coldren, Appl.Phys.Lett. 55 (1989) 2002.

[7] M.M.Dignam and J.E.Sipe, Phys.Rev.Lett. 64 (1990) 1797.

[8] M.M.Dignam and J.E.Sipe, Phys.Rev.B 43 (1991) 4097.

[9] G.H.Wannier, Phys.Rev. 52 (1937) 191.

[10] R.Zimmermann, Phys.Stat.Sol.(b) 135 (1986) 681.

[11] See, for instance, G.Barton, Elements of Green's Functions and Propagation (Clarendon Press, 1989) pp51-56.

[12] E.E.Mendez, F.Agulló-Rueda and J.M.Hong, Phys.Rev.Lett. 60 (1988) 2426. 